\title{
Nasal Manifestations in Granulomatosis with Polyangiitis: A Case Report and Review of the Literature
}

\author{
Massimo Ralli, PhD'*; Vittorio D’ Aguanno, MD²; Vincenzo Falasca, MD²; Rosaria Turchetta, MD²;Antonio Greco, MD²; \\ Marco de Vincentiis, MD'
}

'Department of Oral and Maxillofacial Sciences, Sapienza University of Rome, Viale del Policlinico 155, 00I 86, Rome, Italy

${ }^{2}$ Department of Sense Organs, Sapienza University of Rome, Viale del Policlinico 155, 00 I86, Rome, Italy

\section{"Corresponding author}

Massimo Ralli, PhD

Department of Oral and Maxillofacial Sciences, Sapienza University of Rome.Viale del Policlinico 155, 00 I86, Rome, Italy; Tel. +39 0649976808;

E-mail: massimo.ralli@uniromal.it

\section{Article information}

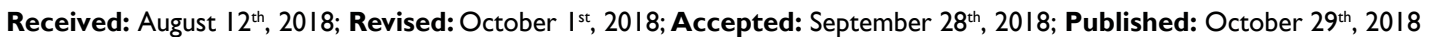

\section{Cite this article}

Ralli M, D' Aguanno V, Falasca V, Turchetta R, Greco A, de Vincentiis M. Nasal manifestations in granulomatosis with polyangiitis: A case report and review of the literature Otolaryngol Open J. 2018; 4(2): 22-25. doi: I0. I7I40/OTLOJ-4-I49

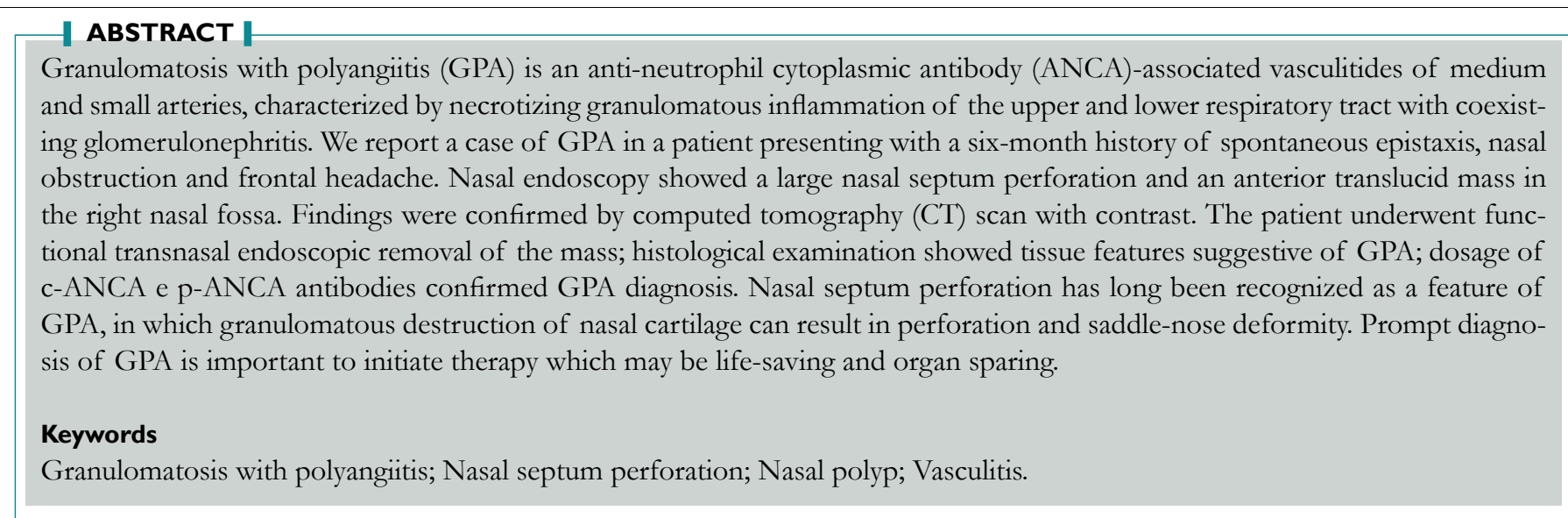

\section{INTRODUCTION |}

ranulomatosis with polyangiitis (GPA), previously known as

JWegener granulomatosis, is an anti-neutrophil cytoplasmic antibodies (ANCA)-associated vasculitides of medium and small arteries, characterized by necrotizing granulomatous inflammation of the upper (ear, nose, throat) and lower (lung) respiratory tract with coexisting glomerulonephritis (renal systems). Untreated generalized GPA is a serious disease that may lead to an irreversible organ damage as renal or lung failure. ${ }^{1-3}$ The estimated incidence of GPA in Europe is 5-10 cases per 1 million population. ${ }^{4}$

The disease is equally distributed between males and females and affects people at any age, but the most common age of presentation of GPA is the sixth and seventh decade of life. In $80-95 \%$ of the patient, the presenting symptoms of GPA are otorhinolaryngological manifestations. ${ }^{5}$
We report a case of GPA in a patient presenting with a six-month history of spontaneous epistaxis, nasal obstruction and frontal headache and evidence at physical examination of a polypoid mass in the nasal fossae and an asymptomatic perforation of the nasal septum.

\section{CASE REPORT}

A 38-year-old women presented to the Otolaryngology Department of our University reporting a six-month history of spontaneous epistaxis, nasal obstruction and frontal headache. Otolaryngologic physical examination through nasal endoscopy showed an anterior perforation of the nasal septum and a polypoid translucid mass in the anterior right nasal cavity. The patient reported to habitually smoke 20 cigarettes/die for 20-years and denied previous nasal surgery, nasal trauma, chronic use of nasal decongestants, and assumption of cocaine. 
The patient performed maxillofacial computed tomography (CT) scan with contrast that confirmed the presence of a soft density polypoid mass occupying the anterior portion of right nasal fossa associated with cartilaginous nasal septum perforation (Figure 1). General laboratory exams were within normal range. Inflammatory marker assays for C-reactive protein and erythrocyte sedimentation rate were within normal limits. In the clinical suspicion of a malignant condition, the patient Figure I: Axial Computed Tomography (CT) Scan without Contrast Showing a Soft
Density Polypoid Mass Occupying the Anterior Portion of Right Nasal Fossa,Associated
with Cartilaginous Nasal Septum Perforation. The Tissue Arises from the Cartilagineal
Portion of Nasal Septum and is in Contact with the Lateral Wall of the Nose. Minimal
Signs of Body Erosion are Present. Around Structure and Maxillary Sinus are Not
Involved.

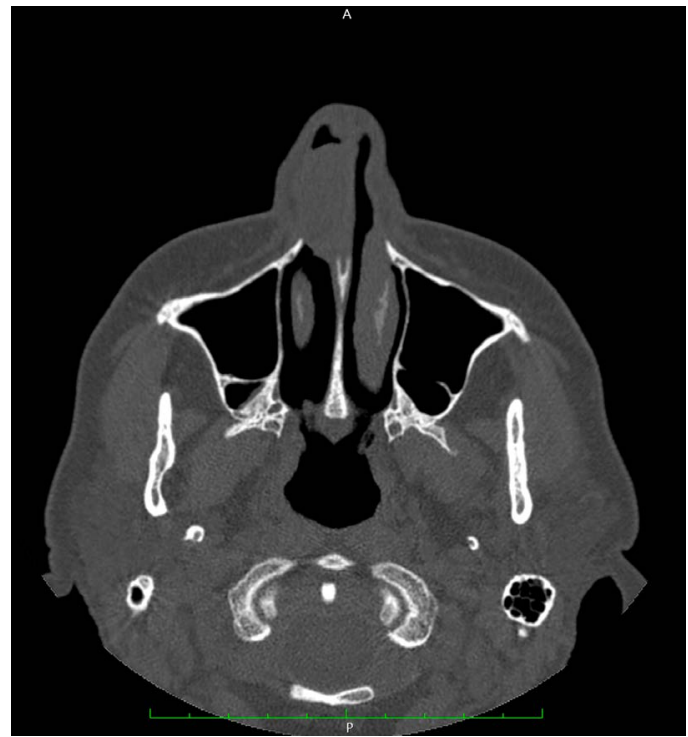

Underwent a trans-nasal endoscopic excision of the mass under general anesthesia. Histological examination showed mixed inflammatory cells T-lymphocytes (CD $\left.3^{+}, \mathrm{CD} 56^{\circ}\right)$, histio-

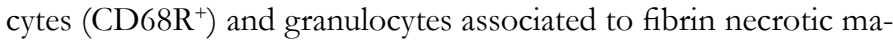
terial and vascular structures with elastic lamina fragmentation and lumen obliteration. Due to the histological suspicion of a vasculitides, Dosage of c-ANCA e p-ANCA antibodies revealed the presence of GPA (the complete immunological tests are reported in Table 1). The post-operative course was uneventful, and the

\begin{tabular}{|ccc}
\hline \multicolumn{3}{|l}{ Table I. Patient's Blood Test } \\
\hline & Patient value & Normal range \\
\hline White blood cell $\left(\times 10^{3} / \mathrm{mm}^{3}\right)$ & 8,46 & $4,40-\mathrm{I} \mathrm{I}, 3$ \\
\hline Platelet $\left(\times 10^{3} / \mathrm{mm}^{3}\right)$ & 306 & $150-450$ \\
\hline Haemoglobin $(\mathrm{g} / \mathrm{dL})$ & 14 & $12,5-15,5$ \\
\hline Prothrobin time $(\mathrm{INR})$ & 0,93 & $0,80-1,20$ \\
\hline c-ANCA $(\mathrm{U})$ & 55,80 & $<20$ \\
\hline P-ANCA $(\mathrm{U})$ & $<3,20$ & $<20$ \\
\hline VES $(\mathrm{mm} / \mathrm{h})$ & 4,0 & $<35$ \\
\hline PRC $(\mathrm{mg} / \mathrm{L})$ & 3 & $0-5$ \\
\hline ENA $(\mathrm{U})$ & $<0,50$ & $<20$ \\
\hline & &
\end{tabular}

patient was sent to the Immunologic Department of our hospital. No signs of recurrence of the diseases were observed one year after surgery.

\section{DISCUSSION}

Granulomatosis with polyangiitis (GPA), previously known as Wegener granulomatosis, is an idiopathic vasculitis involving medium and small arteries.

Among patients with GPA, $80 \%-95 \%$ of patients of develop head and neck manifestations during their life $^{5}$ and often otorhinolaryngological symptoms are the presenting and sole signs of the disease.

In case of a GPA developing only in Head and Neck region, this condition is called "limited GPA", differing from more advanced form, named "generalized GPA", characterized by systemic vasculitis with renal and/or pulmonary involvement and systemic symptoms such as fever and asthenia. "Limited GPA" phenotype is more likely to affect young female patients, with a recurrent behavior and often non-compliant to medical therapy. ${ }^{6}$ Nasal involvement has long been recognized as a feature of "Limited GPA" and usually starts in the septum area supplied by Kiesselbach plexus and then spreads to the paranasal sinuses. ${ }^{7}$

In our case, patient's symptoms are related to sinonasal region, without systemic involvement. Nasal endoscopy reveled a large nasal septum perforation and an anterior translucid mass in the right nasal fossa. Presence of a translucid mass in the middle meatus is often related to an inflammatory disease like nasal polyps, although concomitant presence of a nasal septum perforation (NSP) is unusual.

In case of a NPS is essential the exclusion of cocaine previous use, due to the possibility of an induced destruction of nasal septum. ${ }^{8}$

NPS has long been recognized as a common feature of autoimmune disorders. As in our case, granulomatous destruction of nasal cartilage can result in nasal septum perforation and may develop in advantaged stages to a saddle-nose deformity., ${ }^{910}$ A recent systematic review of the existing literature from Guntupalli et al found that GPA and relapsing polychondritis are the autoimmune disorders most frequently related with nasal septum perforation and comprises nearly $80 \%$ of the reported cases of autoimmune-related nasal septum perforation cases in the literature (Figure 2). ${ }^{11}$

Proper and early diagnosis of GPA is essential for imminent therapy implementation and allows avoiding irreversible organ damage. ${ }^{5}$ Diagnosis of GPA may be challenging given various clinical manifestations, and may be difficult to distinguish from neoplastic, infectious and inflammatory etiologies., ${ }^{212}$ Differential diagnosis must be done with malignant conditions as Natural Killer T-cell lymphoma; infectious disease such as tuberculosis, aspergillosis and leishmaniasis and inflammatory conditions as sarcoidosis. 


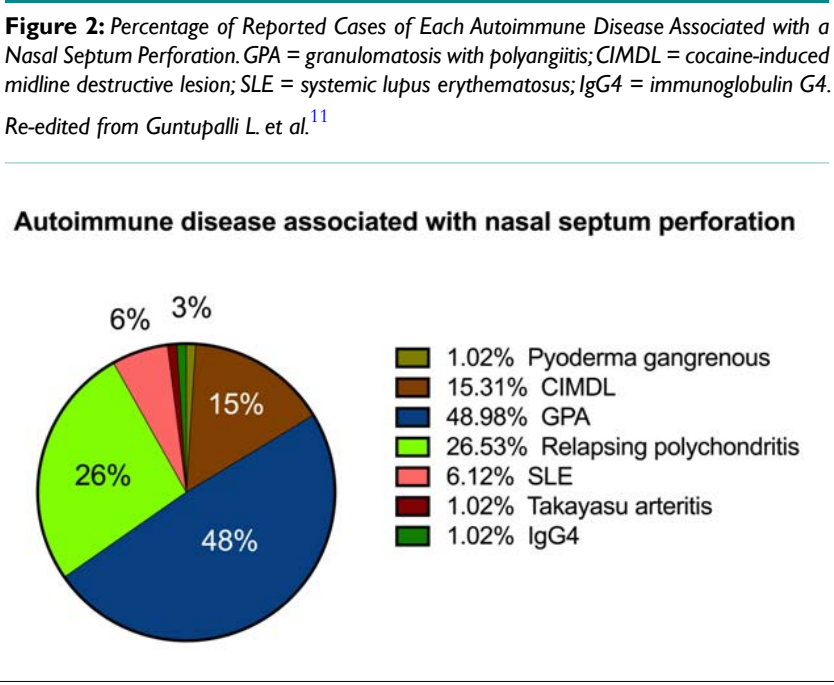

Currently in clinical practice diagnosis is usually based on presence of distinctive ANCA and biopsy of affected organ. ${ }^{13}$ In the clinical suspicion of GPA, ANCA testing is essential for GPA diagnosis. c-ANCA (cytoplasmic anti-neutrophil cytoplasmic antibody) are quite specific for GPA, while p-ANCA (peri-nuclear-anti-neutrophil cytoplasmic antibody) may be associated with other autoimmune conditions like inflammatory bowel disease, rheumatoid arthritis, autoimmune liver disease. During active phases of the disease, the sensitivity and specificity of testing for c-ANCA are $91 \%$ and $99 \%$, respectively. ${ }^{14}$ In the generalized GPA, ANCA are elevated in $90 \%-95 \%$ of patients, whereas in the limited stage of GPA in the ear-nose-throat region, positive levels of c-ANCA may occur in only $46 \%-70 \%$ of patients. ${ }^{15}$ Cytoplasmic localization of ANCA (c-ANCA) is $90-95 \%$ sensitive in acute generalized GPA and $60 \%$ sensitive in early or localized disease. ${ }^{16}$

Biopsy is usually performed when ANCA testing is non-diagnostic or uncertain. Moreover in "limited GPA", the role of the biopsy increases, because predictive value of c-ANCA is lower. ${ }^{13}$ Histological finding of GPA is characterized by specific abnormalities, such as vasculitis, granulomas, giant cells and necrosis. The sensitivity and specificity of tissue biopsy varies depending on the site of active disease. In patients with nasal involvement, nasal biopsy should be considered early in the evaluation, although an high rate of false negative is reported, comparing with biopsy at other affected sites, thus repeated nasal biopsies are often required.

Although nasal manifestations are quite specific for GPA in head and neck, the occurrence of GPA in other head and neck sites is not unusual. Audiovestibular symptoms are often underestimated, and may occur in $8 \%$ to $65 \%$ of patients with GPA. ${ }^{17}$ Typically auditory symptoms are characterized by sensorineural hearing-loss ${ }^{18}$ although conductive hearing loss may also occur in cases of GPA involving the middle ear or Eustachian tube dysfunction. ${ }^{17}$ Audiometric patterns of GPA are typically flat and may coexist with age-related high-frequency loss. Moreover sudden sensorineural hearing loss may also be a presenting symptom of GPA and hearing loss is often a marker of a worsening of disease that may address to a specific treatment. ${ }^{17}$ (Other Head and Neck subsites may be involved in GPA, a summary of most common feature is reported in Table 2).

Awareness of this condition is essential for clinical otorhinolaryngologist, considering the various clinical manifestations of GPA and a prompt diagnosis is essential to initiate specific therapy and avoid renal or lung failure. ${ }^{19}$

\begin{tabular}{|c|c|c|}
\hline H\&N subsite & Frequency & Symptoms \\
\hline Nose and sinuses & $\begin{array}{l}\text { Frequent } \\
(>85 \%)\end{array}$ & $\begin{array}{l}\text { Nasal obstruction and discharge. } \\
\text { Reduction of smell, cacosmia, epiphora. } \\
\text { Perforations of nasal septum with "saddle nose" deformity. }\end{array}$ \\
\hline Ear & $\begin{array}{l}\text { Frequent } \\
(19 \%-61 \%)\end{array}$ & $\begin{array}{l}\text { Frequent: Otitis media secondary to Eustachian Tube dysfunction } \\
\text { Sensorineural hearing loss in } 5 \%-31 \% \text { of GPA patients. } \\
\text { Rare: Sudden sensorineural hearing loss, vertigo, chronic mastoiditis facial paralysis secondary } \\
\text { to bone destruction. } \\
\text { External ear manifestations as erythematous or ulcerated lesions. }\end{array}$ \\
\hline Salivary glands & rare & Parotid or submandibular glands may be swollen and sore. \\
\hline Larynx & rare & $\begin{array}{l}\text { Subglottic stenosis in childhood. } \\
\text { Larynx ulcers. }\end{array}$ \\
\hline Oral cavity & rare & $<20$ \\
\hline
\end{tabular}

\section{CONCLUSION}

GPA is an anti-neutrophil cytoplasmic antibodies (ANCA)-associated vasculitides of medium and small arteries. GPA may present with an isolated nasal involvement. Immunological disorders like GPA should be suspected in patients presenting nasal septum perforation without history of other possible causes of nasal perforation, such as previous cocaine use or nasal surgery. Serological exams and histological features are essential to confirm GPA diagnosis, especially in a case of a "limited GPA". Prompt diagnosis of GPA is important to initiate specific therapy which may be life-saving and organ sparing.

\section{CONSENT}

The patient provided written informed consent for the publication of any associated data and accompanying images. 


\section{FUNDING}

The authors declare that they have no conflicts of interest.

\section{CONFLICTS OF INTEREST |}

The authors declare that they have no conflicts of interest.

\section{REFERENCES}

1. Lutalo P.M, D'Cruz DP. Diagnosis and classification of granulomatosis with polyangiitis (aka Wegener's granulomatosis). J Autoimmun. 2014; 94(8): 48-49. doi: 10.1016/j.jaut.2014.01.028

2. Comarmond C, Cacoub P. Granulomatosis with polyangiitis (Wegener): Clinical aspects and treatment. Autoimmun Rev. 2014. 13(11): 1121-1125. doi: 10.1016/j.autrev.2014.08.017

3. Csernok E, Gross WL. Current understanding of the pathogenesis of granulomatosis with polyangiitis (Wegener's). Expert Rev Clin Immunol. 2013; 9(7): 641-648. doi: 10.1586/1744666X.2013.811052

4. Watts RA, Lane S, Scott D, et al. Epidemiology of vasculitis in Europe. Ann Rheum Dis. 2001. 60(12): 1156-1157. doi: 10.1136\%2Fard.60.12.1156a

5. Srouji IA, Andrews P, Edwards C, Lund VJ. Patterns of presentation and diagnosis of patients with Wegener's granulomatosis: ENT aspects. J Laryngol Otol. 2007. 121(7): 653-658. doi: 10.1017/ S0022215106005032

6. Holle JU, Gross WL, Holl-Ulrich K, et al. Prospective long-term follow-up of patients with localised Wegener's granulomatosis: Does it occur as persistent disease stage? Ann Rheum Dis. 2010. 69(11): 1934-1939. doi: 10.1136/ard.2010.130203

7. Lloyd G, Lund VJ, Beale T, Howard D. Rhinologic changes in Wegener's granulomatosis. J Laryngol Otol. 2002. 116(7): 565-569. doi: 10.1258/002221502760132737

8. Trimarchi M, Sinico RA, Teggi R, et al. Otorhinolaryngological manifestations in granulomatosis with polyangiitis (Wegener's). Autoimmun Rev. 2013; 12(4): 501-505. doi: 10.1016/j.autrev.2012.08.010

9. Kühn D, Hospowsky C, Both M, Hey M, Laudien M. Manifesta- tion of granulomatosis with polyangiitis in head and neck. Clin Exp Rheumatol. 2018; 111(2): 78-84.

10. Diamantopoulos II, NS Jones. The investigation of nasal septal perforations and ulcers. J Laryngol Otol. 2001. 115(7): 541-544.

11. Guntupalli L, Patel K, Faraji F, et al. Autoimmune-related nasal septum perforation: A case report and systematic review. Allergy Rhinol (Providence). 2017; 8(1): 40-44. doi: 10.2500\%2Far.2017.8.0191

12. Grygiel-Górniak B, Limphaibool N, Perkowska K, Puszczewicz M. Clinical manifestations of granulomatosis with polyangiitis: Key considerations and major features. Postgrad Med. 2018; 130(7): 581-596. doi: 10.1080/00325481.2018.1503920

13. Wojciechowska J, Krajewski W, Krajewski P, Kręcicki T. Granulomatosis with polyangiitis in otolaryngologist practice: A review of current knowledge. Clin Exp Otorbinolaryngol. 2016; 9(1): 8-13. doi: $10.21053 /$ ceo.2016.9.1.8

14. Rao JK, Weinberger M, Oddone EZ, et al. The role of antineutrophil cytoplasmic antibody (c-ANCA) testing in the diagnosis of Wegener granulomatosis. A literature review and meta-analysis. Ann Intern Med. 1995. 123(12): 925-32.

15. Borner U, Landis BN, Banz Y, et al. Diagnostic value of biopsies in identifying cytoplasmic antineutrophil cytoplasmic antibody-negative localized Wegener's granulomatosis presenting primarily with sinonasal disease. Am J Rhinol Allergy. 2012; 26(6): 475-480. doi: 10.2500/ajra.2012.26.3825

16. Weeda LW, Coffey SA. Wegener's granulomatosis. Oral Maxillofac Surg Clin North Am. 2008; 20(4): 643-649.

17. Ralli M, D'Aguanno V, Stadio AD, et al. Audiovestibular symptoms in systemic autoimmune diseases. J Immunol Res. 2018. doi: $10.1155 / 2018 / 5798103$

18. Takagi D, Nakamaru Y, Maguchi S, Furuta Y, Fukuda S. Otologic manifestations of Wegener's granulomatosis. Laryngoscope. 2002; 112(9): 1684-1690. doi: 10.1097/00005537-200209000-00029

19. Holle JU, Moosig F, Gross WL, et al. [Therapy of vasculitides: According to recommendations of the European League Against Rheumatism (EULAR) and European Vasculitis Study Group (EUVAS)]. Internist (Berl). 2011; 52(6): 671-681. doi: 10.1007/ s00108-010-2772-5 